\title{
Path integral optimization from Hartle-Hawking wave function
}

\author{
Jan Boruch $\odot,{ }^{1}$ Pawel Caputa $\odot,{ }^{1}$ and Tadashi Takayanagi $\odot^{2,3,4}$ \\ ${ }^{1}$ Faculty of Physics, University of Warsaw, ulica Pasteura 5, 02-093 Warsaw, Poland \\ ${ }^{2}$ Yukawa Institute for Theoretical Physics, Kyoto University, Kitashirakawa Oiwakecho, \\ Sakyo-ku, Kyoto 606-8502, Japan \\ ${ }^{3}$ Inamori Research Institute for Science, 620 Suiginya-cho, Shimogyo-ku, Kyoto 600-8411, Japan \\ ${ }^{4}$ Kavli Institute for the Physics and Mathematics of the Universe, University of Tokyo, \\ Kashiwa, Chiba 277-8582, Japan
}

(Received 1 December 2020; accepted 4 February 2021; published 25 February 2021; corrected 30 August 2022)

We propose a gravity dual description of the path integral optimization in conformal field theories [Caputa et al., Phys. Rev. Lett. 119, 071602 (2017)], using Hartle-Hawking wave functions in anti-de Sitter spacetime. We show that the maximization of the Hartle-Hawking wave function is equivalent to the path integral optimization procedure. Namely, the variation of the wave function leads to a constraint, equivalent to the Neumann boundary condition on a bulk slice, whose classical solutions reproduce metrics from the path integral optimization in conformal field theories. After taking the boundary limit of the semiclassical Hartle-Hawking wave function, we reproduce the path integral complexity action in two dimensions, as well as its higher- and lower-dimensional generalizations. We also discuss an emergence of holographic time from conformal field theory path integrals.

DOI: 10.1103/PhysRevD.103.046017

\section{INTRODUCTION}

The AdS/CFT correspondence [1] provides us with a surprising relation between gravity and quantum manybody systems. Nevertheless, the fundamental mechanism of how it works so well is still not understood. This problem is one of the main obstacles when we try to extend the holographic duality to more general spacetimes, including realistic universe. One interesting idea, pioneered in [2], toward uncovering the basic mechanism behind AdS/CFT is to relate the emergent anti-de Sitter (AdS) geometries to tensor networks such as Multi-scale Entanglement Renormalization Ansatz [3-5] or more general ones [6-8], realizing emergence of spacetimes from quantum entanglement [9]. In particular, this tensor network interpretation beautifully explains the geometric calculation of entanglement entropy in AdS/CFT [10,11]. Refer to, e.g., [12-19] for further developments in this direction. However, these tensor network approaches have been limited to toy models on discrete lattices and precise relations between them and genuine AdS/CFT is not clear. See also recent attempts directly from AdS/CFT [20-23].

Published by the American Physical Society under the terms of the Creative Commons Attribution 4.0 International license. Further distribution of this work must maintain attribution to the author(s) and the published article's title, journal citation, and DOI. Funded by SCOAP .
On the other hand, the path integral optimization [24,25], that we will now review, provides a useful framework that describes tensor networks for conformal field theories (CFTs) in terms of path integrals. We take the Euclidean $\mathbb{R}^{2}$ coordinates $(\tau, x)$ and denote all fields in the CFT by $\Phi(\tau, x)$. The ground state wave functional $\Psi_{\mathrm{CFT}}[\Phi(x)]$ at the time slice $\tau=0$ is defined by the path integral

$$
\begin{aligned}
& \Psi_{\mathrm{CFT}}[\Phi(x)] \\
& =\int \prod_{-\infty<\tau \leq 0, x}[D \tilde{\Phi}(\tau, x)] e^{-S_{\mathrm{CFT}}[\tilde{\Phi}]} \delta(\tilde{\Phi}(0, x)-\Phi(x)),
\end{aligned}
$$

where $S_{\mathrm{CFT}}$ is the action of the 2D CFT.

In the path integral optimization, we deform the metric of our 2D space on which we perform the path integral as follows:

$$
d s^{2}=e^{2 \phi(\tau, x)}\left(d \tau^{2}+d x^{2}\right) .
$$

We take $e^{2 \phi(\tau, x)}=1 / \epsilon^{2}$ for the flat metric of $\mathbb{R}^{2}$ used in the original path integral that computes $\Psi_{\mathrm{CFT}}[\Phi(x)]$, where $\epsilon$ is a UV regularization scale (i.e., lattice constant) when we discretize path integrals of quantum fields into those on a lattice. The curved space metric is interpreted as a choice of discretization such that there is a single lattice site per a unit area.

Let us write the wave functional obtained from the path integral on the curved space (2) as $\Psi_{\mathrm{CFT}}^{\phi}[\Phi(x)]$. If we impose the boundary condition 


$$
e^{2 \phi(0, x)}=\frac{1}{\epsilon^{2}} \equiv e^{2 \phi_{0}}
$$

this wave functional is proportional to the one $\Psi_{\mathrm{CFT}}[\Phi(x)]\left(=\Psi_{\mathrm{CFT}}^{\phi_{0}}[\Phi(x)]\right)$ for the flat space $\mathbb{R}^{2}$ since the CFT is invariant up to the Weyl anomaly

$$
\Psi_{\mathrm{CFT}}^{\phi}[\Phi(x)]=e^{S_{L}[\phi]-S_{L}\left[\phi_{0}\right]} \cdot \Psi_{\mathrm{CFT}}^{\phi_{0}}[\Phi(x)] .
$$

Here $S_{L}$ is the Liouville action [26]

$$
S_{L}[\phi]=\frac{c}{24 \pi} \int_{-\infty}^{\infty} d x \int_{-\infty}^{0} d \tau\left[\left(\partial_{x} \phi\right)^{2}+\left(\partial_{\tau} \phi\right)^{2}+\mu e^{2 \phi}\right]
$$

and $c$ is the central charge of the 2D CFT. The assumption of the discretization, that one unit area of the metric (2) has a single lattice site, fixes the values of $\mu$ to $\mu=1$ [24]. Nevertheless, it is useful to keep this cosmological constant parameter for later purpose.

Relation (4) guarantees that the quantum state is still the same CFT vacuum $|0\rangle$ for any choice of the metric (2) as long as the boundary condition (3) is satisfied. Since the potential term in (5) originates from the UV divergence and we consider $S_{L}$ as a bare action, it should dominate over the kinetic term when we take the UV cutoff to infinity. This is realized when

$$
\left(\partial_{i} \phi\right)^{2} \ll e^{2 \phi} \quad(i=x, \tau) .
$$

The idea of path integral optimization is to coarse grain the discretization as much as possible, which makes computational costs minimal, while keeping the correct answer to the final wave functional. This path integral optimization is performed by minimizing the functional $S_{L}[\phi]$ [24]. This is because we want to minimize the overall factor of the wave functional, which is proportional to $e^{S_{L}[\phi]}$ as in (4). Even though the overall factor does not affect physical quantities in quantum mechanics, this estimates the number of repetitions of numerical integrals when we discretize the required path integral into lattice calculations whose regularization is specified by the metric (2). Therefore, the Liouville action $S_{L}($ at $\mu=1)$ was identified with a measure of computational complexity [27], called the path integral complexity [24] (refer to [28-31] for connections to circuit complexity). The minimization procedure picks up the most efficient discretization of path integral which leads to the correct vacuum state. This method was generalized to various CFT setups in [32-34] and used to compute entanglement of purification in 2D CFTs [35], which was recently verified numerically in [36].

For the vacuum, the minimization is performed by solving the Liouville equation $\left(\partial_{x}^{2}+\partial_{\tau}^{2}\right) \phi=\mu e^{2 \phi}$ with the boundary condition (3), leading to the solution

$$
e^{2 \phi(\tau, x)}=\frac{1}{(\sqrt{\mu} \tau-\epsilon)^{2}}, \quad(-\infty<\tau \leq 0)
$$

The solution at $\mu=1$ is the genuine optimized one, which means the minimization of the original Liouville action. The choice $\mu<1$ may be regarded as partially optimized solution where the UV cutoff length scale is taken to be larger, while the choice $\mu>1$ is not allowed as this corresponds to fine graining the cutoff scale more than the current lattice spacing.

The observation that (7) coincides with the time slice of a three-dimensional AdS geometry $\left(\mathrm{AdS}_{3}\right)$, leads to the main implication that the path integral optimization can explain an emergent AdS geometry purely from CFT [24]. The discretized path integral takes the form of a (nonunitary) tensor network and its relation to AdS geometry can be regarded as a path integral version of the conjectured interpretation of AdS/CFT as tensor networks. However, a direct connection between the path integral optimization and $\mathrm{AdS} / \mathrm{CFT}$ has remained an open problem.

Another subtle issue is that, in the solution (7), we find $\left(\partial_{i} \phi\right)^{2}$ and $e^{2 \phi}$ are of the same order, which does not satisfy the criterion (6). This suggests that the path integral optimization using the Liouville action is qualitative and can have finite cutoff corrections.

Moreover, it has not been clear how to promote the classical Liouville theory equivalent to the above path integral optimization to a quantum Liouville theory. Indeed, it was found in [24] that to properly reproduce the correct gravity metric dual to a primary state with $1 / c$ corrections, we need to replace a classical Liouville theoretic result with a quantum Liouville theoretic one. This is because the path integration $\int[D \phi] e^{S_{L}[\phi]}$ in (4) does not make sense as it is not bounded from below. Instead the quantum Liouville theory is defined by the path integral $\int[D \phi] e^{-S_{L}[\phi]}$. In other words, we cannot get the minimization as a saddle point approximation of path integrals and a derivation of path integral optimization from AdS/CFT remained a challenge.

In this paper, we would like to resolve these important issues by introducing a gravity dual description in terms of a Hartle-Hawking wave function which evolves from the $\mathrm{AdS}$ boundary. This corresponds to the gravity action in the

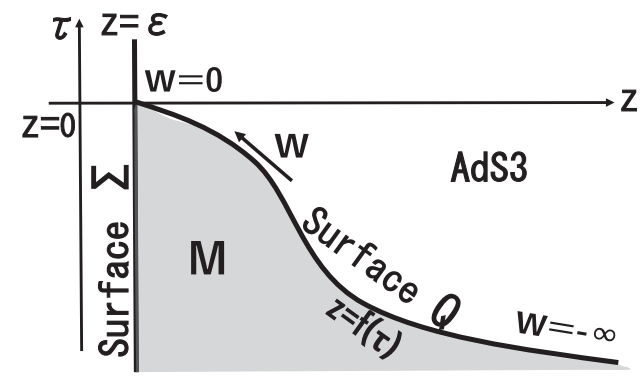

FIG. 1. The on-shell action in the colored region $M$ provides a gravity evaluation of the Hartle-Hawking wave function. 
shaded region in Fig. 1, assuming the Euclidean Poincaré $\operatorname{AdS}_{d+1}$ geometry

$$
d s^{2}=\frac{d z^{2}+d \tau^{2}+\sum_{i=1}^{d-1} d x_{i}^{2}}{z^{2}}
$$

\section{HARTLE-HAWKING WAVE FUNCTION WITH BOUNDARY}

Consider a Hartle-Hawking wave function [37] in an $\operatorname{AdS}_{d+1}$, denoted by $\Psi_{H H}\left[g_{a b}\right]$, which is a functional of the metric $g_{a b}$ on a surface $Q$. Respecting the timelike boundary in AdS, we impose an initial condition on the AdS boundary $\Sigma$ given by $z=\epsilon$ and $\tau<0$. Then we consider a path integral of Euclidean gravity from this asymptotic boundary to the surface $Q$ which extends from $z=\epsilon$ and $\tau=0$ toward the bulk as depicted in Fig. 1. Focusing on translational invariant setups for simplicity, we assume the diagonal form metric on $Q$

$$
d s^{2}=e^{2 \phi}\left(d w^{2}+\sum_{i=1}^{d-1} d x_{i}^{2}\right),
$$

where $w$ is a function of $\tau$. This way, we can write the Hartle-Hawking wave function as $\Psi_{H H}[\phi(w, x)]$, defined by

$$
\Psi_{H H}[\phi]=\int\left[D g_{\mu \nu}\right] e^{-I_{G}[g]} \delta\left(\left.g_{a b}\right|_{Q}-e^{2 \phi} \delta_{a b}\right),
$$

where $I_{G}$ is the $d+1$-dimensional gravity action

$I_{G}=-\frac{1}{16 \pi G_{N}} \int_{M} \sqrt{g}(R-2 \Lambda)-\frac{1}{8 \pi G_{N}} \int_{Q \cup \Sigma} \sqrt{h} K$.

We implicitly imposed a boundary condition on $\Sigma$. Even though we choose that of pure AdS dual to the CFT vacuum, in principle, we can consider $\Psi_{H H}[\phi(w, x)]$ corresponding to excited states of a CFT (see below).

Finally, we propose to identify the metric (9) with (2) (in $d=2$ case) and, after setting $w=\tau$, we argue that the optimization procedure corresponds to the maximization of $\Psi_{H H}[\phi]$ with respect to $\phi$. This maximization can be understood naturally when we consider an evaluation of correlation function as

$$
\left\langle O_{1} O_{2} \ldots\right\rangle=\int[D \phi]\left|\Psi_{H H}[\phi]\right|^{2} O_{1} O_{2} \ldots
$$

by applying the saddle point approximation. In this way, the maximization of a Hartle-Hawking wave function works well even in the presence of quantum fluctuations of $\phi$, as opposed to the minimization of $e^{S_{L}[\phi]}$. Indeed, below, we will show that $\Psi_{H H}[\phi]$ is proportional to $e^{-S_{L}[\phi]}$ up to finite cutoff corrections.
It will also be useful to add a tension term on the brane $Q$ as in the AdS-boundary CFT (BCFT) [38] (we assume $T<0$ below)

$$
I_{T}[h]=\frac{T}{8 \pi G_{N}} \int_{Q} \sqrt{h}
$$

and define one parameter family of deformed HartleHawking wave functions as follows:

$\Psi_{H H}^{(T)}[\phi]=\int\left[D g_{\mu \nu}\right] e^{-I_{G}[g]-I_{T}\left[e^{2 \phi}\right]} \delta\left(\left.g_{a b}\right|_{Q}-e^{2 \phi} \delta_{a b}\right)$.

The standard Hartle-Hawking wave functions are obtained by setting $T=0$. We can regard $T$ as a chemical potential or Legendre transformation for the area of $Q$, acting on Hartle-Hawking wave functions. As we will see, the tension term plays the role of the cosmological constant term in the Liouville action. More importantly, since the maximum of $\Psi_{H H}^{(T)}[\phi]$ corresponds to a family of surfaces $Q$ in AdS parametrized by the tension $T$, we will observe that $T$ plays the role of an emergent holographic time.

\section{EVALUATION OF $\Psi_{H H}^{(T)}[\phi]$}

Let us evaluate $\Psi_{H H}^{(T)}[\phi]$ using semiclassical approximation, i.e., as an on-shell gravity action

$$
\left.\Psi_{H H}^{(T)}[\phi] \simeq e^{-I_{G}[g]-I_{T}[h]}\right|_{\text {on shell }} .
$$

For simplicity we assume that the metric (9) has a translational invariance in the $x$ direction. For a given choice of such a metric $e^{2 \phi}$, we find a surface $Q$ specified by the profile

$$
z=f(\tau) .
$$

Then the semiclassical evaluation of (10) will give the value of the Hartle-Hawking wave function.

Note that the above construction assumes that a gravity solution which calculates the Hartle-Hawking wave function is given by a subregion in a Poincaré AdS geometry. The metric (9) obtained in this way covers all possible metrics on $Q$ for $d=2$ with the condition (3) because all solutions to the vacuum Einstein equation are locally equivalent to the Poincare $\mathrm{AdS}_{3}$. However, for $d>2$, the above construction covers only a part of the metrics on the $d$-dimensional surface $Q$. Nevertheless, this ansatz includes a class of metric we want, as we will see below. It is also useful to note that we can extend our targets to general metrics by directly solving the Einstein equation.

The $w$ coordinate and the metric in (9) is found as

$$
e^{\phi}=f^{-1}, \quad \frac{d \tau}{d w}=\sqrt{1-\dot{f}^{2}},
$$


where $\dot{f}$ means $\partial_{w} f$. We set $w=0$ at $\tau=0$ and thus we have $e^{-\phi}=\epsilon$ at $w=0$. Then, the on-shell action on $M$, defined by $\epsilon<z<f(\tau)$ (see $M$ in Fig. 1) is evaluated in terms of the coordinate $w$ as follows:

$$
\begin{aligned}
I_{G}+I_{T}= & -\frac{(d-1) V_{x} L_{\tau}}{8 \pi G_{N} \epsilon^{d}}+\frac{(d-1) V_{x}}{8 \pi G_{N}} \int d w e^{d \phi} G(\dot{\phi}) \\
& -\frac{V_{x}}{8 \pi G_{N}}\left[e^{(d-1) \phi} \arcsin \left(\dot{\phi} e^{-\phi}\right)\right]_{-\infty}^{0},
\end{aligned}
$$

where $V_{x}$ and $L_{\tau}$ are infinite volumes in $x$ and $\tau$ directions and $G$ is the following function bounded from below:

$$
G(\dot{\phi})=\sqrt{1-e^{-2 \phi} \dot{\phi}^{2}}+\dot{\phi} e^{-\phi} \arcsin \left(\dot{\phi} e^{-\phi}\right)+\frac{T}{d-1} .
$$

When we neglect the finite cutoff corrections assuming (6), we can approximate the on-shell action (18) as a quadratic action of $\phi$. In $d=2$, this expansion yields

$$
\begin{aligned}
I_{G}+I_{T} \simeq & \frac{c}{12 \pi} \int d x d w\left[\frac{1}{2} \dot{\phi}^{2}+(1+T) e^{2 \phi}-\epsilon^{-2}\right] \\
& -\frac{c}{12 \pi} \int d x \frac{\theta_{0}}{\epsilon},
\end{aligned}
$$

where $\theta_{0}$ is the value of $\arcsin \left(\dot{\phi} e^{-\phi}\right)$ at $w=0$. We can cancel $\theta_{0}$ dependence by adding the corner term [39] localized on $\Sigma \cap Q$. This reproduces the "path integral complexity" action [24] $I\left[\phi, \phi_{0}\right]=S_{L}[\phi]-S_{L}\left[\phi_{0}\right]$ with the correct coefficient of the kinetic term (remember we assumed $\left.\partial_{x} \phi=0\right)$. Note that the above gravity computation (18) gives the correct finite cutoff corrections to the Liouville action and thus provides a full answer to the path integral optimization. The same is true in higher dimensions. Notice that, unusual from the perspective of complexity, properties $I\left[g_{1}, g_{2}\right]=-I\left[g_{2}, g_{1}\right]$ and $I\left[g_{1}, g_{2}\right]+$ $I\left[g_{2}, g_{3}\right]=I\left[g_{1}, g_{3}\right]$ become manifest from the gravity action with boundaries.

\section{SOLUTIONS}

Now we would like to maximize the Hartle-Hawking wave function (15). This is performed by taking a variation of the on-shell action (18) with respect to $\phi$, leading to

$$
\frac{e^{-2 \phi}\left(\ddot{\phi}+(d-1) \dot{\phi}^{2}\right)-d}{\sqrt{1-e^{-2 \phi} \dot{\phi}^{2}}}=\frac{d}{d-1} T .
$$

By imposing the boundary condition

$$
\left.e^{2 \phi}\right|_{w=0}=\frac{1}{\epsilon^{2}},
$$

we obtain the solution to (21) when $T<0$ as follows:

$$
e^{2 \phi}=\frac{1}{\left(\sqrt{1-\frac{T^{2}}{(d-1)^{2}}} w-\epsilon\right)^{2}} .
$$

This corresponds to the following surface in (8):

$$
z=\epsilon+\sqrt{1-\frac{T^{2}}{(d-1)^{2}}} \frac{(d-1)}{T} \tau .
$$

For the solution (23), the on-shell action is evaluated as

$$
I_{G}+I_{T}=-\frac{(d-1) V_{x} L_{\tau}}{8 \pi G_{N} \epsilon^{d}}-\frac{V_{x} \theta_{0}}{\epsilon^{d-1}}
$$

where $\sin \theta_{0}=\sqrt{1-\frac{T^{2}}{(d-1)^{2}}}$.

Previously we observed that the maximization of the Hartle-Hawking wave function is given by minimizing the integral of (19), i.e., the Liouville action plus finite cutoff corrections. On the other hand, the original path integral optimization is proposed to be simply given by the minimization of Liouville action. However, it is possible that this apparent deviation arises because the regularization scheme is different between the CFT and gravity formation, though they are actually equivalent. Though we do not have any definite argument for this, the fact that both give the same profile of optimized solution may imply this equivalence. Indeed if we set

$$
\mu=1-\frac{T^{2}}{(d-1)^{2}},
$$

then the solution (23) is equal to that from the path integral optimization (7). Remember that changing $\mu$ from $\mu=0$ to $\mu=1$ means that we gradually increase the amount of optimization. In the gravity dual, this corresponds to changing the tension from $T=-(d-1)$ to $T=0$ which tilts the surface $Q$ from the asymptotic boundary $\Sigma$ to the time slice $\tau=0$.

Note also that the Eq. (21) for $\phi$ is equivalent to imposing the Neumann boundary condition on $Q$

$$
K_{a b}-K h_{a b}=-T h_{a b},
$$

which is imposed in the AdS/BCFT construction [38].

Let us finally stress that, in higher dimensions $d>2$ there has not been a complete formulation of path integral optimization known until now as we do not know a higher-dimensional counterpart of the Liouville action. Remarkably, our approach using the Hartle-Hawking wave function gives the full answer to this question for CFTs with gravity duals. 


\section{EXCITED STATES}

Furthermore, we consider a family of Euclidean Bañados-Teitelboim-Zanelli-type metrics in three dimensions

$$
d s^{2}=\left(r^{2}-r_{h}^{2}\right) d \tau^{2}+\frac{d r^{2}}{r^{2}-r_{h}^{2}}+r^{2} d x^{2},
$$

where $r_{h}^{2}=M-1$ can be positive or negative depending on the mass of the excitation. We can repeat our analysis of $\Psi_{H H}^{T}$ for region $\frac{1}{f(\tau)} \leq r \leq \frac{1}{\epsilon}$, where $r=1 / f(\tau)$ and $r=1 / \epsilon$ describe the surface $Q$ and the asymptotic boundary $\Sigma$, respectively. Our action has the same form as (18) for $d=2$ and

$$
\begin{aligned}
G(\dot{\phi})= & \sqrt{1-e^{-2 \phi}\left(\dot{\phi}^{2}+r_{h}^{2}\right)}+T \\
& +\dot{\phi} e^{-\phi} \arcsin \left(\frac{\dot{\phi} e^{-\phi}}{\sqrt{1-r_{h}^{2} e^{-2 \phi}}}\right) .
\end{aligned}
$$

Variation with respect to $\phi$ yields $K_{Q}=2 T$ and is again equivalent to the Neumann condition (27). For negative tension, we can solve it by

$$
e^{2 \phi}=\frac{r_{h}^{2}}{\left(1-T^{2}\right) \sin ^{2}\left(r_{h}\left(w-c_{1}\right)\right)} .
$$

This family of solutions precisely matches those in the path integral optimization [24] via the identification (26). For $r_{h}=(2 \pi) / \beta$ and $w$ on the strip, we reproduce our optimal geometry for the thermofield double state dual to the time slice of the Einstein-Rosen bridge [40]. For $r_{h}^{2}=-(1-M)$ we reproduce excited states from the optimization for primary operators in 2D CFT, i.e., conical singularity geometries, including the finite size vacuum. In all these examples we choose $c_{1}$ such that (3) is fulfilled at each boundary. Moreover, we can verify (either by explicit computation or using the Wheeler-DeWitt equation) that our solutions (7), (23), and (30) have constant negative curvature that depends on $\mu$ [or $T$ via (26)].

Last but not least, we can test our prescription in the context of Jackiw-Teitelboim (JT) gravity dual to the Sachdev-Ye-Kitaev (SYK) model [41-45]. In this case, it turns out that it is advantageous to introduce the tension on $Q$ by coupling to the dilaton $\Phi$

$$
\begin{aligned}
I_{J T}+I_{T_{\Phi}}= & -\left[\int_{M} \sqrt{g} \Phi(R+2)+2 \int_{\partial M} \sqrt{h} \Phi K\right] \\
& -\Phi_{0} \chi(M)+2 T_{\Phi} \int_{Q} \sqrt{h} \Phi
\end{aligned}
$$

with $\chi(M)$ being the Euler characteristic of our region $M$. As an example, we can take the analogous 2D solution

$$
d s^{2}=\left(r^{2}-r_{h}^{2}\right) d \tau^{2}+\frac{d r^{2}}{r^{2}-r_{h}^{2}}, \quad \Phi=\Phi_{b} r .
$$

Defining $M$ bounded by $\Sigma$ at $r=r_{0} \rightarrow \infty$ and $Q$ by $r=1 / f(\tau)$, with induced metric

$$
d s^{2}=e^{2 \phi} d w^{2}, \quad e^{\phi}=f^{-1},
$$

we can derive a JT analog of (29), show that the saddle point equation is the Neumann boundary condition and find that its solution is given by (30) with $T \rightarrow T_{\Phi}$. Moreover, in the UV limit of small $\dot{\phi}$ our action reproduces the effective Schwarzian description of the SYK model with the symmetry breaking term. This confirms the validity of our approach in all dimensions. We performed analogous studies for higher-dimensional black holes as well as examples of Lorentzian spacetimes and details will be presented in [46].

\section{CONCLUSIONS AND DISCUSSION}

In this paper, we showed that the path integral optimization corresponds to the maximization of the HartleHawking wave function, which is a functional of the metric (9) on a surface $Q: \operatorname{Max}_{\phi}\left[\Psi_{H H}[\phi]\right]$. This HartleHawking wave function with the boundary condition (22), describes an evolution from an initial condition set by the AdS boundary, dual to the target CFT state for which we perform the path integral optimization. An important requirement is that the surface $Q$ ends on the AdS boundary and this gives the boundary condition (22). Owing to this requirement, we can calculate CFT quantities such as correlations functions from an inner product of the Hartle-Hawking wave functional as in (12), whose saddle point approximation gives the maximization of $\Psi_{H H}[\phi]$.

Furthermore, we generalize our correspondence to nontrivial parameters: $\mu$ in Liouville theory and tension $T$ in gravity, related by (26). In the path integral optimization $\mu$ controls the scale up to which we perform the coarse graining and this optimization procedure is maximized at $\mu=1$. On the gravity side, this scale is fixed by the tension term (13), which plays the role of a chemical potential to the area of the surface $Q$. Even though the original Hartle-Hawking wave function does not have any time dependence, "time" emerges by considering the $T$-dependent one: $\Psi_{H H}^{(T)}[\phi]$, where the tension plays a role of external field. From the CFT side, time emerges as the scale $\mu$ of the optimization, related to $T$ via (26). Indeed, using the optimized solution (23) or (30), we can write the full $\mathrm{AdS}_{d+1}$ space as follows:

$$
d s^{2}=\frac{d \mu^{2}}{4 \mu^{2}(1-\mu)}+e^{2 \phi}\left(d w^{2}+d x_{i}^{2}\right) .
$$

Note that this foliation is a special case of the York time [47] (refer to [48] for an interesting interpretation of York time from AdS/CFT). It will be a very important future direction to 
derive the genuine AdS/CFT itself by starting from the purely CFT analysis of path integral optimization. We believe that this emergent time observation provides us with an important clue in this direction.

\section{ACKNOWLEDGMENTS}

We are grateful to Sumit Das, Diptarka Das, Dongsheng Ge, Jacek Jezierski, Masamichi Miyaji, and Onkar Parrikar for useful discussions. T. T. is supported by Grant-in-Aid for JSPS Fellows No. 19F19813. T. T. is supported by the Simons Foundation through the "It from Qubit" collaboration. T. T. is supported by Inamori Research Institute for Science and World Premier International Research Center Initiative (WPI Initiative) from the Japan Ministry of Education, Culture, Sports, Science and Technology (MEXT). T. T. is supported by JSPS Grant-in-Aid for Scientific Research (A) No. 16H02182. T. T. is also supported by JSPS Grantin-Aid for Challenging Research (Exploratory) No. 18K18766. P. C. and J. B. are supported by National Agency for Academic Exchange, Poland Polish Returns 2019 Grant No. PPN/PPO/2019/1/00010/U/0001 and NCN Sonata Bis 9 Grant No. 2019/34/E/ST2/00123.
[1] J. M. Maldacena, The large N limit of superconformal field theories and supergravity, Adv. Theor. Math. Phys. 2, 231 (1998); Int. J. Theor. Phys. 38, 1113 (1999).

[2] B. Swingle, Entanglement renormalization and holography, Phys. Rev. D 86, 065007 (2012).

[3] G. Vidal, Class of Quantum Many-Body States That Can Be Efficiently Simulated, Phys. Rev. Lett. 101, 110501 (2008); Entanglement Renormalization, Phys. Rev. Lett. 99, 220405 (2007).

[4] G. Evenbly and G. Vidal, Tensor Network Renormalization, Phys. Rev. Lett. 115, 180405 (2015); Tensor Network Renormalization Yields the Multiscale Entanglement Renormalization Ansatz, Phys. Rev. Lett. 115, 200401 (2015).

[5] J. Haegeman, T. J. Osborne, H. Verschelde, and F. Verstraete, Entanglement Renormalization for Quantum Fields, Phys. Rev. Lett. 110, 100402 (2013).

[6] X. L. Qi, Exact holographic mapping and emergent spacetime geometry, arXiv:1309.6282.

[7] F. Pastawski, B. Yoshida, D. Harlow, and J. Preskill, Holographic quantum error-correcting codes: Toy models for the bulk/boundary correspondence, J. High Energy Phys. 06 (2015) 149.

[8] P. Hayden, S. Nezami, X. L. Qi, N. Thomas, M. Walter, and Z. Yang, Holographic duality from random tensor networks, J. High Energy Phys. 11 (2016) 009.

[9] M. Van Raamsdonk, Building up spacetime with quantum entanglement, Gen. Relativ. Gravit. 42, 2323 (2010).

[10] S. Ryu and T. Takayanagi, Holographic Derivation of Entanglement Entropy from AdS/CFT, Phys. Rev. Lett. 96, 181602 (2006).

[11] V.E. Hubeny, M. Rangamani, and T. Takayanagi, A covariant holographic entanglement entropy proposal, J. High Energy Phys. 07 (2007) 062.

[12] C. Beny, Causal structure of the entanglement renormalization ansatz, New J. Phys. 15, 023020 (2013).

[13] M. Nozaki, S. Ryu, and T. Takayanagi, Holographic geometry of entanglement renormalization in quantum field theories, J. High Energy Phys. 10 (2012) 193.

[14] M. Miyaji and T. Takayanagi, Surface/state correspondence as a generalized holography, Prog. Theor. Exp. Phys. 2015, 073B03 (2015).
[15] B. Czech, L. Lamprou, S. McCandlish, and J. Sully, Integral geometry and holography, J. High Energy Phys. 10 (2015) 175.

[16] B. Czech, L. Lamprou, S. McCandlish, and J. Sully, Tensor networks from kinematic space, J. High Energy Phys. 07 (2016) 100.

[17] A. Milsted and G. Vidal, Tensor networks as path integral geometry, arXiv:1807.02501.

[18] A. Milsted and G. Vidal, Geometric interpretation of the multi-scale entanglement renormalization ansatz, arXiv: 1812.00529

[19] A. Jahn, Z. Zimborás, and J. Eisert, Tensor network models of AdS/qCFT, arXiv:2004.04173.

[20] T. Takayanagi, Holographic spacetimes as quantum circuits of path-integrations, J. High Energy Phys. 12 (2018) 048.

[21] M. Van Raamsdonk, Building up spacetime with quantum entanglement II: It from BC-bit, arXiv:1809.01197.

[22] N. Bao, G. Penington, J. Sorce, and A. C. Wall, Beyond toy models: Distilling tensor networks in full AdS/CFT, J. High Energy Phys. 11 (2019) 069.

[23] N. Bao, G. Penington, J. Sorce, and A. C. Wall, Holographic tensor networks in full AdS/CFT, arXiv:1902.10157.

[24] P. Caputa, N. Kundu, M. Miyaji, T. Takayanagi, and K. Watanabe, Anti-de Sitter Space from Optimization of Path Integrals in Conformal Field Theories, Phys. Rev. Lett. 119, 071602 (2017); Liouville action as path-integral complexity: From continuous tensor networks to AdS/CFT, J. High Energy Phys. 11 (2017) 097.

[25] M. Miyaji, T. Takayanagi, and K. Watanabe, From path integrals to tensor networks for AdS/CFT, Phys. Rev. D 95, 066004 (2017).

[26] A. M. Polyakov, Quantum geometry of bosonic strings, Phys. Lett. 103B, 207 (1981).

[27] L. Susskind, Computational complexity and black hole horizons, Fortschr. Phys. 64, 24 (2016); A. R. Brown, D. A. Roberts, L. Susskind, B. Swingle, and Y. Zhao, Holographic Complexity Equals Bulk Action?, Phys. Rev. Lett. 116, 191301 (2016).

[28] B. Czech, Einstein Equations from Varying Complexity, Phys. Rev. Lett. 120, 031601 (2018). 
[29] P. Caputa and J. M. Magan, Quantum Computation as Gravity, Phys. Rev. Lett. 122, 231302 (2019).

[30] H. A. Camargo, M. P. Heller, R. Jefferson, and J. Knaute, Path Integral Optimization as Circuit Complexity, Phys. Rev. Lett. 123, 011601 (2019).

[31] J. Erdmenger, M. Gerbershagen, and A. L. Weigel, Complexity measures from geometric actions on Virasoro and Kac-Moody orbits, J. High Energy Phys. 11 (2020) 003.

[32] Y. Sato and K. Watanabe, Does boundary distinguish complexities?, J. High Energy Phys. 11 (2019) 132.

[33] P. Caputa and I. MacCormack, Geometry and complexity of path integrals in inhomogeneous CFTs, J. High Energy Phys. 01 (2021) 027.

[34] A. Bhattacharyya, P. Caputa, S. R. Das, N. Kundu, M. Miyaji, and T. Takayanagi, Path-integral complexity for perturbed CFTs, J. High Energy Phys. 07 (2018) 086.

[35] P. Caputa, M. Miyaji, T. Takayanagi, and K. Umemoto, Holographic Entanglement of Purification from Conformal Field Theories, Phys. Rev. Lett. 122, 111601 (2019).

[36] H. A. Camargo, L. Hackl, M. P. Heller, A. Jahn, T. Takayanagi, and B. Windt, Entanglement and complexity of purification in $(1+1)$-dimensional free conformal field theories, arXiv:2009.11881 [Phys. Rev. Research (to be published)].

[37] J. B. Hartle and S. W. Hawking, Wave function of the Universe, Adv. Ser. Astrophys. Cosmol. 3, 174 (1987).

[38] T. Takayanagi, Holographic Dual of BCFT, Phys. Rev. Lett. 107, 101602 (2011); M. Fujita, T. Takayanagi, and E.
Tonni, Aspects of AdS/BCFT, J. High Energy Phys. 11 (2011) 043.

[39] G. Hayward, Gravitational action for space-times with nonsmooth boundaries, Phys. Rev. D 47, 3275 (1993).

[40] J. M. Maldacena, Eternal black holes in anti-de Sitter, J. High Energy Phys. 04 (2003) 021.

[41] A. Kitaev, A simple model of quantum holography, in Proceedings at KITP, 2015.

[42] S. Sachdev and J.-w. Ye, Gapless Spin Fluid Ground State in a Random, Quantum Heisenberg Magnet, Phys. Rev. Lett. 70, 3339 (1993).

[43] J. Maldacena and D. Stanford, Remarks on the Sachdev-YeKitaev model, Phys. Rev. D 94, 106002 (2016).

[44] J. Maldacena, D. Stanford, and Z. Yang, Conformal symmetry and its breaking in two dimensional nearly anti-deSitter space, Prog. Theor. Exp. Phys. 2016, 12C104 (2016).

[45] K. Jensen, Chaos in $\mathrm{AdS}_{2}$ Holography, Phys. Rev. Lett. 117, 111601 (2016).

[46] J. Boruch, P. Caputa, and T. Takayanagi (to be published).

[47] J. W. York, Jr., Role of Conformal Three Geometry in the Dynamics of Gravitation, Phys. Rev. Lett. 28, 1082 (1972).

[48] A. Belin, A. Lewkowycz, and G. Sárosi, Complexity and the bulk volume, a New York time story, J. High Energy Phys. 03 (2019) 044.

Correction: Support information in the Acknowledgments section was incomplete and has been fixed. 